\title{
Diagnostic value of computerized tomography venography in detecting stenosis and occlusion of subclavian vein and superior vena in chronic renal failure patients
}

\author{
Banafsheh Bakhshoude ${ }^{1}$, Hassan Ravari², Gholam Hosein Kazemzadeh ${ }^{3}$, Masoud Pezeshki Rad ${ }^{4}$
}

${ }^{1}$ M.D., Resident of Radiology, Department of Radiology, Faculty of Medicine, Mashhad University of Medical Sciences, Mashhad, Iran

${ }^{2}$ M.D., Associate Professor, Department of Vascular Surgery, Imam Reza Hospital, Mashhad University of Medical Sciences, Mashhad, Iran

${ }^{3}$ M.D., Associate Professor, Department of Vascular Surgery, Vascular and Endovascular Surgery Research Center, Imam Reza Hospital, Mashhad University of Medical Sciences, Mashhad, Iran

${ }^{4}$ M.D., Associate Professor, Department of Radiology, Vascular and Endovascular Surgery Research Center, Mashhad University of Medical Sciences, Mashhad, Iran

\section{Type of article: Original}

\begin{abstract}
Introduction: Currently, venography is the standard diagnostic method to examine veins before implementing access, which is invasive in nature. Computerized tomography venography (CTV) can simultaneously indicate deep and superficial venous systems in the upper extremity and their relation to the surrounding anatomical structures; however, its diagnostic value in the detection of central venous disease has yet to be defined. The aim of this study was to determine the diagnostic value of CT venography compared to venography in the diagnosis of stenosis and the occlusion of subclavian veins and the superior vena in renal failure patients.

Methods: This cross-sectional study was conducted from January to September 2015 on patients with chronic renal failure undergoing upper extremity venography at the Radiology Department of Imam Reza Hospital in Mashhad, Iran. We excluded patients with catheters in their jugular and subclavian vein routes, venous hypertension with reverse-function fistula, or sensitivity to contrast agents. Several factors, including age, gender, catheterization record in jugular and subclavian veins, and fistula record in the upper extremity, as well as clinical symptoms consisting of edema, dermatitis, and ulcers in these organs, were recorded in the corresponding form. Then, the patients consecutively underwent indirect venography and CT venography and traces of stenosis (more than $50 \%$ ) or complete occlusion in the subclavian vein and superior vena were recorded. The data were analyzed using SPSS software by the chi-squared test, and sensitivity, specificity, and positive and negative predictive values were calculated by means of MedCalc Online, version 16.2.

Results: The study was conducted on 40 patients ( 26 males and 14 females) with a mean age of $46.7 \pm 10.4$ years. In this study, 58 subclavian veins, as well as 32 superior vena cava, were studied. The results showed that the diagnostic value of CTV in the detection of subclavian stenosis had a sensitivity and a specificity of 88.2 and $97.5 \%$, respectively. Moreover, in the superior vena cava, the greatest CTV diagnostic sensitivity and specificity was related to vein stenosis detection (sensitivity $=88.8 \%$; specificity $=100 \%$ ).

Conclusion: It seems that the CTV, based on its high sensitivity, specificity, and predictive value, can be used as an efficient tool in the study of stenosis in subclavian veins and superior vena in patients undergoing venography Keywords: Venography, CT venography, Hemodialysis, Renal failure, Central venous
\end{abstract}

\section{Corresponding author:}

Masoud Pezeshki Rad, Associate Professor, Department of Radiology, Vascular and Endovascular Surgery Research Center, Mashhad University of Medical Sciences, Mashhad, Iran.

Tel: +98.9151131491, Email: PezeshkiradM@mums.ac.ir Received: January 10, 2016, Accepted: February 22, 2016, Published: August 2016 iThenticate screening: February 22, 2016, English editing: July 16, 2016, Quality control: August 02, 2016 (C) 2016 The Authors. This is an open access article under the terms of the Creative Commons Attribution-NonCommercialNoDerivs License, which permits use and distribution in any medium, provided the original work is properly cited, the use is non-commercial and no modifications or adaptations are made. 


\section{Introduction}

Chronic renal failure (CRF) refers to irreversible impairment of renal function, which eventually leads to end-stage renal disease (ESRD) (1). CRF has experienced a growing trend in recent years, and its annual growth in Iran is about $11 \%$ according to the statistics by the Special Diseases Center of the Ministry of Health and Treatment. The CRF also has an incidence rate of 250 cases per one million people $(2,3)$. According to the latest available statistics, more than half of people with CRF are currently under permanent hemodialysis treatment, and more than 150,000 dialyses are performed monthly in Iran $(3,4)$. In ESRD patients, before embedding access, including artificial vein or arteriovenous fistula, hemodialysis is performed through temporary catheterization of the subclavian and jugular veins. Based on previous studies, such temporary catheterization leads to the incidence of stenosis or occlusion in these veins, but, since the stenosis process occurs slowly and there is sufficient time for collateral vessel formation, symptoms related to venous stenosis and the incidence of venous hypertension symptoms are not apparent until the artificial vein or arteriovenous fistula is embedded (5-8). In this case, the failure to detect stenosis or occlusion in patients who are candidates for the implantation of an artificial vein or arteriovenous fistula, in addition to imposing exorbitant costs on the patient and the Health Department, causes venous hypertension and its complications (2-9). Currently, venography is the standard diagnostic method to evaluate the veins prior to embedding (10). This evaluation method is invasive and requires a separate catheterization of suitable veins in the area of each hand. Direct injection of the contrast agent has the risk of developing thrombophlebitis and destruction of the veins used for the access. It is sometimes difficult to technically indicate the deep and superficial venous systems at the same time and to interpret the resulting images $(10,11)$. In this method, only those veins in the draining route of the contrast agent can be studied; other veins are not visible. Recently, several studies have described the great diagnostic power of CTV in the detection of lower extremity deep venous thrombosis (DVT) $(12,13)$. In most of these studies, the sensitivity of CTV in comparison with the gold diagnostic standard for DVT is equal to $100 \%$, and its specificity is reported to be over $93 \%(12-16)$. However, the results obtained by some recent studies have shown a significant association between the CTV and venography findings in the diagnosis of central vein stenosis and occlusion $(17,18)$. However, to date, no studies have explained the diagnostic power of CTV in detecting stenosis and the occlusion of central veins, and further studies are required due to the routine use of CTV to examine veins prior to access embedding in patients undergoing hemodialysis. Moreover, due to the capability of CT scan's threedimensional images that simultaneously show the deep and superficial venous systems in the upper extremity and their relationship with adjacent anatomical structures, CTV can be used as a road map for the next interventional or surgical procedures $(17,19)$. Therefore, the aim of this study was to evaluate the diagnostic value of CTV in detecting stenosis and occlusion in subclavian veins and superior vena in patients with renal failure.

\section{Material and Methods}

\subsection{Design and setting}

This cross-sectional study was conducted on CRF patients undergoing upper extremity venography at the Radiology Department at Imam Reza Hospital in Mashhad, Iran from January to September 2015. This study included patients who had histories of jugular or subclavian vein catheterization and were candidates for the implantation of an artificial vein or fistula. Exclusion criteria included the incidence of venous hypertension or sensitivity to the contrast agent.

\subsection{Sampling}

The samples were evaluated and selected from the initial population of all patients undergoing venography who had the inclusion criteria and did not have the exclusion criteria during the implementation period.

\subsection{Measurement tool}

\subsubsection{Checklist:}

A checklist, including age, gender, and clinical symptoms consisting of the presence or absence of swelling, dermatitis, ulcers, and collateral vessels in the upper extremity, was recorded for all the patients by a radiology resident. All of the patients underwent CTV and then venography in their proximal upper extremity veins. Furthermore, the incidence of stenosis (more than $50 \%$ in the veins) and occlusion in the subclavian veins and superior vena was recorded separately in each patient's checklist based on the results of each of the two methods.

2.3.2. Venography:

Upper extremity venography was performed by a radiology assistant under the supervision of an Associate Professor of Interventional Radiology. In venography with the standard protocol, after fastening a tourniquet around the arm having a superficial vein punctured with a 18-gauge needle and with the hand in the supine position and briefly abducted from the body, 30-50 cc low osmolality nonionic contrast material (ultravist, $300 \mathrm{mg} / \mathrm{dl}$ ) was injected with 
a flow rate of 4 to $8 \mathrm{cc}$ per second using an injector. After the radiology resident performed the above procedures, the images were evaluated and approved by the corresponding professor.

\subsection{3. $C T V$ :}

Before performing CTV, the patients were asked to take out their clothes so that even the least probable pressure on the superficial veins was eliminated. The patients were investigated in the supine position and with their arms briefly abducted from the body, and, then, the CTV tests were conducted using an MDCT scanning device (16 Neosoft). An 18-20 gauge catheter was put through in the right or left side using a suitable antecubital vein, and, then, $2 \mathrm{ml} / \mathrm{kg}$ of the non-ionic contrast material (iopromide, Ultravist 370, Schering Company) was injected mechanically with an injector at the rate of $2.5 \mathrm{ml} / \mathrm{s}$. After the injection of the contrast agent, $50 \mathrm{cc}$ of normal saline were injected. The CTV images were recorded three minutes after the start of the injection of the contrast material from the neck base to the front wrists of both sides. CTV has a number of scanning parameters for the 16-MDCT scanner, including a $0.75 \times 16 \mathrm{~mm}$ detector collimation, a 1.5 pitch, a $1 \mathrm{~mm}$ slice thickness, a $0.7 \mathrm{~mm}$ reconstruction interval, a $120 \mathrm{kVp}$ $\mathrm{X}$-ray tube voltage, as well as a $150 \mathrm{mAs}$ effective tube current. The scan ranged from the base of the neck to the end of the hands. After performing the CTV, pivotal, thin-section images were transferred to the three-dimensional (3D) reconstruction software, and the 3D reconstruction techniques were conducted. The CTV three-dimensional images were examined indirectly by a radiology assistant under the supervision of an experienced radiology specialist.

\subsection{Ethical consideration}

This study was approved by the Ethics Committee at Mashhad University of Medical Sciences (Code: 922465). All of the patients were informed of the content of this research project as well as its benefits and complications prior to being included in the study, and all signed the consent form. Moreover, the patients' privacy and dignity were observed and respected while implementing the project. The data obtained from the patients also were coded into the statistical analysis program, and the results were published in the form of an overall conclusion.

\subsection{Statistical analyses}

The demographic and paraclinical data of the patients were entered into the computer, and statistical analysis was performed using SPSS 17.0 for Windows (SSPS, Inc., Chicago, IL, USA). First, the normal distribution of the data was examined. Given the normal distribution of the data, the quantitative data were expressed as mean $\pm \mathrm{SD}$ and $\mathrm{P}<$ 0.05 was considered significant. To examine the relationship between the findings of CTV and venography, both the chi-squared test and, when necessary, Fisher's exact test, were put to use. Furthermore, other variables of the CTV findings, including sensitivity, specificity, and positive and negative predictive values were calculated based on venography using Medcalc software (version 16.2).

\section{Results}

\subsection{Baseline characteristics}

During the nine-month period of the study, among the $73 \mathrm{CRF}$ patients who went to the Hospital to have venography of their central veins performed, 45 patients who met the inclusion criteria and were included in the study. However, from this initial sample, five patients were excluded due to the incidence of venous hypertension (n $=3$ ) and sensitivity to the contrast agent $(n=2)$. Ultimately, the study was conducted with 40 patients $(26$ males and 14 females) with a mean age of $46.7 \pm 10.4$ years. The reason for referral of all the patients to perform venography was the evidence of dysfunction of jugular or subclavian vein catheterization. In this study, the most common findings obtained from the examination of the patients were swelling of the upper extremity $(30 \%)$ and stasis dermatitis (5\%), and no specific findings were observed in the other patients after the clinical examination. In this study, 58 subclavian veins and 32 superior vena cava were studied. Stenosis was the most common finding in the venography of the subclavian veins and superior vena (29.3 and 28.1\%, respectively) (Table 1). However, occlusion was most commonly observed in the CTV in a way that the occlusion incidence was $27.5 \%$ in the subclavian veins and $28.1 \%$ in the superior vena cava (Table 1 ).

Table 1. Central vein stenosis and occlusion frequency in patients in the study separated by the imaging type

\begin{tabular}{|l|l|l|l|l|}
\hline Variables & \multicolumn{2}{|l|}{ Venography } & \multicolumn{2}{l|}{ CT Venography } \\
\hline Vein type & Stenosis & Occlusion & Stenosis & Occlusion \\
\hline Subclavian $(\mathrm{n}=58)$ & $17(29.3 \%)$ & $6(10.3 \%)$ & $16(27.5 \%)$ & $5(86.2 \%)$ \\
\hline Superior vena cava $(\mathrm{n}=32)$ & $9(28.1 \%)$ & $8(25.0 \%)$ & $9(28.1 \%)$ & $6(18.7 \%)$ \\
\hline
\end{tabular}




\subsection{CTV diagnostic value}

The results showed that the highest diagnostic value of CTV in detecting subclavian vein diseases was related to the diagnosis of subclavian stenosis (sensitivity $=88.2 \%$; specificity $=97.5 \%$ ). Moreover, in the superior vena cava, just as in other central veins, the most diagnostic sensitivity and specificity of CTV was related to the detection of stenosis in the vein (sensitivity $=88.8 \%$; specificity $=100 \%)($ Table 2$)$.

Table 2. Diagnostic value of CTV in the detection of central venous diseases

\begin{tabular}{|l|l|l|l|l|l|}
\hline Variables & Vein & Sensitivity & Specificity & $\begin{array}{l}\text { Positive predictive } \\
\text { value (PPV) }\end{array}$ & $\begin{array}{l}\text { Negative predictive } \\
\text { value (NPV) }\end{array}$ \\
\hline Stenosis & Subclavian $(\mathrm{n}=58)$ & 88.2 & 97.5 & 93.7 & 95.2 \\
\cline { 2 - 6 } & $\begin{array}{l}\text { Superior vena cava } \\
(\mathrm{n}=32)\end{array}$ & 88.8 & 100 & 100 & 95.8 \\
\hline Occlusion & Subclavian $(\mathrm{n}=58)$ & 50.0 & 96.1 & 60.0 & 94.3 \\
\cline { 2 - 6 } & $\begin{array}{l}\text { Superior vena cava } \\
(\mathrm{n}=32)\end{array}$ & 62.5 & 95.8 & 83.3 & 88.4 \\
\hline
\end{tabular}

\section{Discussion}

This study was the first study conducted on the evaluation of the CTV diagnostic value in detecting stenosis and occlusion in central subclavian veins and the superior vena, and the results were presented in form of sensitivity, specificity, and positive and negative predictive values of this method in the diagnosis of central subclavian diseases. Based on the results of this study, CTV was regarded as a diagnostic method with high sensitivity and specificity for the diagnosis of central veins in ESRD patients, and, therefore, it can be used at least as a tool for screening central venous diseases. To date, venography has been the standard diagnostic method for the evaluation of central venous status prior to the access implementation in hemodialysis patients despite its high costs, invasive nature, and evaluative power limited to the contrast agent route. However, a recent study showed that CTV also can be used as an alternative method. It is interesting to note that, in all the results obtained in this study, both in the diagnosis of occlusion and stenosis in the both central veins under the study, CTV had a high negative predictive value, so that NPV was obtained as greater than $90 \%$ in most cases. This means that, in case the CTV result shows no diseases in the studied veins, the absence of disease in venography is likely to be at least approximately $90 \%$, which is regarded really good statistics for a new tool. However, the CTV positive predictive value has been more than $90 \%$ in diagnosing central venous diseases, especially in cases of stenosis, meaning that, if a person has a CTV that is suspected of being diseased, there also will be at least a $90 \%$ probability of having stenosis in report of venography. As mentioned earlier, to date, no study has been conducted to investigate the CTV diagnostic power in the detection of stenosis and occlusion of central veins, but several articles have been published in the form of a series of studies on the relationship between the findings of CTV and venography in diagnosing central venous stenosis and occlusion $(17,18)$. The study conducted in 2003 by Kim et al. (17) investigated the role of CTV in the diagnosis and treatment of benign thoracic central venous occlusion, the results of which showed a significant correlation between the degree and rate of venous occlusion in CTV and venography. This study was performed on 18 patients, and its main purpose was not to evaluate the CTV diagnostic power, which makes it totally different from our study. However, most studies have been conducted to evaluate the CTV diagnostic value for venous thrombosis in the upper and lower extremities. In the study conducted by Zontsich et al., the CTV sensitivity and specificity in the diagnosis of upper and lower extremity deep venous thrombosis were obtained as 100 and $96 \%$, respectively, compared to the venography gold standard (20). In another study conducted by Lee et al. to investigate the great saphenous venous insufficiency, the CTV sensitivity and specificity in comparison with venography were obtained as 98.2 and $83.3 \%$, respectively (21). There is no similar study that has produced results that can be used as a comparison with the CTV diagnostic power obtained in our study. However, the reasons for the CTV sensitivity and specificity difference compared to the venography can be summarized as follows. The difference in skill and experience of a CTV examiner can lead to a difference in the CTV accuracy and sensitivity in diagnosing stenosis or occlusion; although in the current study, we interpreted all the CTV results under the supervision of an Associate Professor of Radiology to mitigate the impact of this variable. However, the fat tissue status of the subjects in the study can affect the results. However, we did not investigate the CTV diagnostic value in individuals with different body mass indices in the current study. Moreover, the quality of the device used to perform the CTV also can be influential, and differences in the resolutions of various $\mathrm{CT}$ devices can certainly result in a different accuracy and sensitivity in venous stenosis detection. To date, conventional venography has been used to determine the central venous status in patients with chronic kidney disease (CKD) candidate for access implantation; however, the use of surgical techniques or endovascular interventions, per se, can have the risk of causing the shunt created in central 
veins to be open. Therefore, other vascular assessment methods, such as CTV or Color Doppler Ultrasound, are recommended to be used, at least in cases where there is no certainty of the need for the endovascular intervention (22). In this study, we faced various limitations since it was conducted as a pilot study. The current study was singlecenter in nature, and the CTV results were dependent on the interpretation of the operator. Therefore, further studies should be conducted in different centers to achieve more accurate information regarding the diagnostic value of CTV. Moreover, it is recommended for the diagnostic value of CTV be investigated along with other developing diagnostic methods, such as Color Doppler Ultrasound in future studies.

\section{Conclusions}

According to the high specificity, sensitivity, and predictive values obtained in this study, it seems that the CTV can be used as a non-invasive screening test in diagnosing stenosis of subclavian veins and superior vena. However, further studies are required to enhance the diagnostic accuracy of CTV with respect to vascular occlusion.

\section{Acknowledgments:}

The authors express their thanks to the Vice Chancellor for Research at Mashhad University of Medical Sciences for the financial support that was provided for this study. This manuscript was derived from a Radiology Residency thesis with a project code of 922465 .

\section{Conflict of Interest:}

There is no conflict of interest to be declared.

\section{Authors' contributions:}

All authors contributed to this project and article equally. All authors read and approved the final manuscript.

\section{References:}

1) Messina LE, Claro JA, Nardozza A, Andrade E, Ortiz V, Srougi M. Erectile dysfunction in patients with chronic renal failure. Int Braz J Urol. 2007; 33(5): 673-8. PMID: 17980064.

2) Hojjat M. Hemodialysis adequacy in patients with chronic renal failure. Iranian journal of critical care nursing. 2009; 2(2): 61-6.

3) Raiesifar A, Torabpour M, Mohsenizad P. Causes of chronic renal failure in hemodialysis patients of Abadan. Iranian journal of critical care nursing. 2010; 2(4): 11-12.

4) Asgari MR, Mohammadi E, Fallahi Khoshknab M, Tamadon MR. The perception of chronic renal failure patients from advocacy resources in adjustment with hemodialysis: A qualitative study. Iranian journal of critical care nursing. 2011;3(4): 133-44.

5) Moist LM, Al-Jaishi AA. The upfront risks of vascular access complications. J Am Soc Nephrol. 2013; 24(10): 1509-11. doi: 10.1681/ASN.2013070759. PMID: 23970122, PMCID: 3785286.

6) Siracuse JJ, Gill HL, Epelboym I, Wollstein A, Kotsurovskyy Y, Catz D, et al. Effect of Race and Insurance Status on Outcomes After Vascular Access Placement for Hemodialysis. Ann Vasc Surg. 2014; 28(4): 964-9. doi: 10.1016/j.avsg.2013.10.016. PMID: 24370501.

7) Chan MR, Sanchez RJ, Young HN, Yevzlin AS. Vascular access outcomes in the elderly hemodialysis population: A USRDS study. Semin Dial. 2007; 20(6): 606-10. doi: 10.1111/j.1525-139X.2007.00370.x. PMID: 17991212.

8) Leivaditis K, Panagoutsos S, Roumeliotis A, Liakopoulos V, Vargemezis V. Vascular access for hemodialysis: postoperative evaluation and function monitoring. Int Urol Nephrol. 2014; 46(2): 403-9. doi: 10.1007/s11255-013-0564-2. PMID: 24046176.

9) Ross JR. Insertion of short introducer sheaths into a polyurethane vascular access graft for immediate hemodialysis access. J Vasc Access. 2003; 4(2): 62-7. PMID: 17642062.

10) Les J, Wankowicz Z. Methods of central vascular access for haemodialysis. Anaesthesiol Intensive Ther. 2013; 45(3): 171-6. doi: 10.5603/AIT.2013.0035. PMID: 24092515.

11) Rodriguez CR, Bardon Otero E, Vila Paz ML. Access for starting kidney replacement therapy: vascular and peritoneal temporal access in pre-dialysis. Nefrologia. 2008; 3: 105-12. PMID: 19018747.

12) Peterson DA, Kazerooni EA, Wakefield TW, Knipp BS, Forauer AR, Bailey BJ, et al. Computed tomographic venography is specific but not sensitive for diagnosis of acute lower-extremity deep venous thrombosis in patients with suspected pulmonary embolus. J Vasc Surg. 2001; 34(5): 798-804. doi: 10.1067/mva.2001.118803. PMID: 11700478. 
13) Duwe KM, Shiau M, Budorick NE, Austin JH, Berkmen YM. Evaluation of the lower extremity veins in patients with suspected pulmonary embolism: a retrospective comparison of helical CT venography and sonography. American Journal of Roentgenology. 2000; 175(6): 1525-31. doi: 10.2214/ajr.175.6.1751525. PMID: 11090368.

14) Garg K, Kemp JL, Wojcik D, Hoehn S, Johnston RJ, Macey LC, et al. Thromboembolic disease: comparison of combined CT pulmonary angiography and venography with bilateral leg sonography in 70 patients. AJR Am J Roentgenol. 2000; 175(4): 997-1001. doi: 10.2214/ajr.175.4.1750997. PMID: 11000152.

15) Loud PA, Katz DS, Klippenstein DL, Shah RD, Grossman ZD. Combined CT venography and pulmonary angiography in suspected thromboembolic disease: diagnostic accuracy for deep venous evaluation. AJR Am J Roentgenol. 2000; 174(1): 61-5. doi: 10.2214/ajr.174.1.1740061. PMID: 10628455.

16) Cham MD, Yankelevitz DF, Shaham D, Shah AA, Sherman L, Lewis A, et al. Deep venous thrombosis: detection by using indirect CT venography. The Pulmonary Angiography-Indirect CT Venography Cooperative Group. Radiology. 2000; 216(3): 744-51. doi: 10.1148/radiology.216.3.r00se44744. PMID: 10966705.

17) Kim H, Chung JW, Park JH, Yin YH, Park SH, Yoon CJ, et al. Role of CT venography in the diagnosis and treatment of benign thoracic central venous obstruction. Korean J Radiol. 2003; 4(3): 146-52. doi: 10.3348/kjr.2003.4.3.146. PMID: 14530642, PMCID: 2698080.

18) Kim HC, Chung JW, Yoon CJ, Lee W, Jae HJ, Kim YI, et al. Collateral pathways in thoracic central venous obstruction: three-dimensional display using direct spiral computed tomography venography. J Comput Assist Tomogr. 2004; 28(1): 24-33. doi: 10.1097/00004728-200401000-00004. PMID: 14716228.

19) Bogdan MA, Klein MB, Rubin GD, McAdams TR, Chang J. CT angiography in complex upper extremity reconstruction. J Hand Surg Br. 2004; 29(5): 465-9. doi: 10.1016/j.jhsb.2004.04.006. PMID: 15336751.

20) Zontsich T, Turetschek K, Baldt M. CT-phlebography. A new method for the diagnosis of venous thrombosis of the upper and lower extremities. Der Radiologe. 1998; 38(7): 586-90. doi: 10.1007/s001170050396. PMID: 9738263.

21) Lee W, Chung JW, Yin YH, Jae HJ, Kim SJ, Ha J, et al. Three-Dimensional CT venography of varicose veins of the lower extremity: image quality and comparison with doppler sonography. AJR Am J Roentgenol. 2008; 191(4): 1186-91. doi: 10.2214/AJR.07.3471. PMID: 18806163.

22) Rad MP, Kazemzadeh GH, Ziaee M, Azarkar G. Diagnostic value of color doppler ultrasonography in detecting stenosis and occlusion of central veins in patients with chronic kidney disease. Saudi J Kidney Dis Transp. 2015; 26(2): 279-84. PMID: 25758875 